\title{
ESTUDO DO COMPORTAMENTO DOS MICRO E MACRONUTRIENTES EM MEIO DE CULTIVO DA MICROALGA Scenedesmus sp. PARA PRODUÇÃO DE LIPÍDEOS
}

\author{
W. S. BORGES ${ }^{1}$, B. S. A. ARAÚJO ${ }^{1}$, L. G. MOURA ${ }^{1}$, M.M. RESENDE ${ }^{1}$ e V. L. CARDOSO ${ }^{1}$ \\ ${ }^{1}$ Universidade Federal de Uberlândia, Faculdade de Engenharia Química \\ E-mail para contato: wesley.itb@gmail.com
}

\begin{abstract}
RESUMO - As microalgas apresentam metabolismos distintos. As características metabólicas das microalgas fazem delas uma importante fonte de recursos a serem exploradas. Para o cultivo de microalgas há diversas formulações que contemplam as suas necessidades nutricionais, necessitando de macronutrientes, micronutrientes e vitaminas. A concentração de cada nutriente é exigida em quantidades variadas, dependendo da exigência dos processos metabólitos. O zinco participa da estrutura de enzimas como cofator. Este trabalho teve como objetivo avaliar a produção de lipídeos das células da microalga Scenedesmus sp. em meio Guillard quando há a alteração de seus micro e macronutrientes, otimizando as melhores condições de cultivo. Os resultados mostram que a melhor concentração dos micronutrientes é quando se trabalha com $0,06 \mathrm{~g} / \mathrm{L}$ de $\mathrm{ZnSO}_{4} \cdot 7 \mathrm{H}_{2} \mathrm{O}$ e $0,5 \mathrm{~g} / \mathrm{L} \mathrm{MnCl}_{2} \bullet 4 \mathrm{H}_{2} \mathrm{O}$, chegando a um teor de lipídeos 47,5\% e concentração de biomassa 0,0966g/L. Já a alteração da concentração dos macronutrientes não é viável.
\end{abstract}

\section{INTRODUÇÃO}

Para o cultivo de microalgas há diversas formulações que contemplam as suas necessidades nutricionais, necessitando basicamente de macronutrientes, micronutrientes e vitaminas. Os macronutrientes apresentam a função de constituir as estruturas do meio intracelular, participando do processo de troca de energia e regulando atividades metabólicas, enquanto os micronutrientes participam da estrutura e atividade enzimática. Já as vitaminas, como a tiamina e biotina agem, respectivamente, como coenzima e transportadora de dióxido de carbono (Lourenço, 2006). A concentração de cada nutriente - elemento - é exigida em quantidades variadas, dependendo da exigência dos processos metabólitos das algas. Segundo Lourenço (2006), os nutrientes se dividem em macronutrientes e micronutrientes.

O zinco apresenta papéis metabólicos semelhante aos apresentados para o manganês, que atua na síntese de ácidos graxos. $O$ zinco participa da estrutura de enzimas como co-fator e também é um componente estrutural da enzima anidrase carbônica, que é uma enzima crítica ao transporte e fixação de $\mathrm{CO}_{2}$. Geralmente o zinco é adicionado na forma de $\mathrm{ZnSO}_{4} \cdot 7 \mathrm{H}_{2} \mathrm{O}$, que é uma de suas formas solúveis. O manganês atua como co-fator de enzimas que participam da síntese de ácidos graxos e também do Ciclo de Krebs. Tem importância fundamental no transporte de elétrons do fotossistema, atua na manutenção da estrutura das membranas dos 


\section{9 a 22 de outubro de 2014 \\ Florianópolis/SC}

cloroplastos e também é um componente da enzima superóxido-dismutase, que é a enzima que remove radicais superóxidos tóxicos das células. O manganês é exigido em concentrações mais baixas que as do ferro.

Portanto, este trabalho teve como objetivo avaliar a produção de lipídeos células da microalgas Scenedesmus sp. em meio Guillard quando há a alteração de seus micro e macronutrientes, otimizando as melhores condições de cultivo.

\section{MATERIAL E MÉTODOS 2.1. Microalga}

A cepa da microalga Scenedesmus sp. foi gentilmente cedidas pelo Departamento de Engenharia Química da Universidade Federal do Rio Grande do Sul, em Porto Alegre RS. A microalga Scenedesmus sp. foi coletada na "Lagoa de Cota Cota", em La Paz (Bolívia) e isolada no laboratório de IIDEPROQ - UMSA (Instituto de Investigación y Desarrollo de Procesos Quimicos) da Universidad Mayor de San Andres. A microalga foi mantida em incubadora dotada de fotoperíodo de $12 \mathrm{~h} \mathrm{luz} \mathrm{/} 12 \mathrm{~h}$ escuro, com temperatura a 22,5 $\mathrm{C}^{\circ} \pm 0,5$ e repiques de manutenção feitos a cada 15 dias, posteriormente armazenadas até utilização nos experimentos.

\subsection{Unidade experimental e meio de cultivo}

As microalgas foram mantidas em ambiente climatizado a $23 \pm 1^{\circ} \mathrm{C}$ com iluminação artificial com 3 lâmpadas fluorescentes de $20 \mathrm{~W}$, submetidas a fotoperíodo de 12 horas, cultivadas em reatores verticais em acrílico e reatores abertos em formato de bandejas, com volume útil de 3 litros. Os fotobiorreatores foram construídos seguindo os critérios básicos de cultivo de microalgas de Muñoz e Guieysse (2006): alta elevada eficiência na utilização da energia luminosa, facilidade no controle de aeração, adequado sistema de mistura, facilidade em aumento de escala e reduzido estresse hidrodinâmico das células. Utilizou-se o meio Guillard (1975) modificado, sem a adição de vitaminas.

\subsection{Métodos analíticos}

Determinação do peso seco: Após os cultivos, a amostra foi centrifugada em uma centrífuga modelo Heraeus Megafuge 16, marca Thermo Fischer Scientific, $4800 \mathrm{rpm}$ (correspondente a um campo centrífugo relativo de $7808 \mathrm{~g}$ ) por 5 minutos. A massa da centrifugação foi colocada em béquer previamente pesado e levado para secagem em estufa a $80^{\circ} \mathrm{C}$ por 48 horas. Posteriormente as amostras filtradas foram colocadas em um dessecador por 30 minutos e estas foram pesadas após o período, determinando a biomassa seca final, sendo a concentração de células expressas em g/L (Lourenço, 2006; Gris, 2010).

Teor lipídeos: Utilizou-se o método de Folch (1975) com banho ultrassônico

Estudo da variação das concentrações dos micronutrientes na influência do teor de lipídeos: Foram testadas diferentes concentrações dos micronutrientes no meio de cultivo da microalga 


\section{9 a 22 de outubro de 2014 \\ Florianópolis/SC}

Scendesmus sp. Para cada experimento, todas as soluções-estoque para a preparação do meio de cultivo foram utilizadas, conforme o meio Guillard (1975) modificado. Quando ocorre a variação de um deles, por exemplo no $\mathrm{Na}_{2}$ EDTA, a concentração dos demais mantém-se a mesma já adotada pela literatura. Os experimentos também foram realizados utilizando $10 \%$ do volume de meio de cultivo como inóculo, intensidade luminosa de 5000 lux, aeração de 0,7 vvm nos reatores e volume útil 3 litros. Após o teste com os micronutrientes acima, os micronutrientes que apresentaram algum comportamento relevante na alteração de teor de lipídeos e biomassa foram testados novamente para reproduzir os resultados utilizando as concentrações de $\mathrm{ZnSO}_{4} \cdot 7 \mathrm{H}_{2} \mathrm{O}$ e $\mathrm{MnCl}_{2} \cdot 4 \mathrm{H}_{2} \mathrm{O}$ já otimizadas por meio de um Planejamento Composto Central. As melhores condições da concentração dos micronutrientes foram utilizadas para prosseguir os testes para o estudo com os macronutrientes.

Estudo da variação das concentrações dos macronutrientes: Semelhante aos testes com concentrações dos micronutrientes, os macronutrientes foram submetidos à diferentes concentrações de forma a buscar a melhor performance para a produção lipídica. As condições operacionais foram semelhantes às adotas para o teste com micronutrientes. Nos testes com os macronutrientes, foram utilizadas as concentrações otimizadas dos micronutrientes $\mathrm{ZnSO}_{4} \cdot 7 \mathrm{H}_{2} \mathrm{O}$ e $\mathrm{MnCl}_{2} \cdot 4 \mathrm{H}_{2} \mathrm{O}$ de modo a verificar se algum macronutriente, quando combinado com a melhor concentração dos micronutrientes acima, podem apresentar alguma melhoria na produção lipídica.

\section{RESULTADOS E DISCUSSÃO \\ 3.1. Estudo da variação das concentrações dos micronutrientes na influência no teor de lipídeos}

A Tabela 1 apresenta os resultados obtidos para o teor de lipídeos extraído das células e biomassa seca da microalga Scenedesmus sp. para os experimentos com alteração as concentrações dos micronutrientes $\mathrm{Na}_{2} \mathrm{EDTA}, \mathrm{FeCl}_{3} \cdot \mathrm{H}_{2} \mathrm{O}, \mathrm{CuSO}_{4} \cdot 5 \mathrm{H}_{2} \mathrm{O}$ e $\mathrm{CoCl}_{2} \cdot \mathrm{H}_{2} \mathrm{O}$.

Pelos resultados apresentados na Tabela 1 verifica-se que nos experimentos em que ocorreu alteração nas concentrações de $\mathrm{Na}_{2}$ EDTA e $\mathrm{CuSO}_{4} \cdot 5 \mathrm{H}_{2} \mathrm{O}$ não ocorreu elevação no teor de lipídeos, quando comparados com os do ponto "Normal", em que as concentrações destes no experimento foram as mesmas fornecidas pela literatura. Nota-se que quando há alteração nas concentrações de $\mathrm{Na}_{2}$ EDTA e $\mathrm{CuSO}_{4} \cdot 5 \mathrm{H}_{2} \mathrm{O}$, seja na redução ou aumento da concentração destes, há redução teor de lipídeos. Para a concentração de $\mathrm{FeCl}_{3} \cdot \mathrm{H}_{2} \mathrm{O}$, quando reduz-se sua concentração para a metade, a produção lipídica chega a 30,83\% das células, sendo maior que o valor encontrado de $30,41 \%$ no ponto com as concentrações descritas pela literatura. A Tabela 1 mostra que um aumento de $\mathrm{FeCl}_{3} \cdot \mathrm{H}_{2} \mathrm{O}$ não é bom para a produção de lipídeos, e sim somente se o foco for a produção de biomassa. O estudo sobre a concentração dos micronutrientes foi essencial para verificar qual micronutriente interfere diretamente no teor de lipídeos. Neste ponto, foi selecionado que para as concentrações de $\mathrm{FeCl}_{3} \cdot \mathrm{H}_{2} \mathrm{O}$ e $\mathrm{CoCl}_{2} \cdot \mathrm{H}_{2} \mathrm{O}$ estas poderiam ser reduzidas pela metade, sendo utilizadas soluções estoque com 1,575 g/L de $\mathrm{FeCl}_{3} \cdot \mathrm{H}_{2} \mathrm{O}$ e 0,006 $\mathrm{g} / \mathrm{L}$ de $\mathrm{CoCl}_{2} \cdot \mathrm{H}_{2} \mathrm{O}$, porém caso a alteração fosse somente nestas, independentes 
Tabela. 1 - Resultados para o teor de lipídeos e biomassa para cada experimento com alteração nas concentrações dos micronutrientes.

\begin{tabular}{ccccc}
\hline Micronutriente & Condição & $\begin{array}{c}\text { Concentração na } \\
\text { solução-estoque } \\
(\mathbf{g} / \mathbf{L})\end{array}$ & $\begin{array}{c}\text { Biomassa } \\
(\mathbf{g} / \mathbf{L})\end{array}$ & $\begin{array}{c}\text { Teor de Lipídeos } \\
\text { extraído das células } \\
(\mathbf{\%}) \mathbf{g} / \mathbf{g}\end{array}$ \\
\hline \multirow{2}{*}{$\mathrm{Na}_{2}$ EDTA } & Sem adição & 0 & 0,0767 & 4,35 \\
& Metade & 2,18 & 0,0810 & 4,11 \\
& Normal & $*$ & 0,0739 & 30,41 \\
& Dobro & 8,72 & 0,0533 & 18,75 \\
& Quíntuplo & 21,8 & 0,065 & 5,10 \\
\hline \multirow{2}{*}{$\mathrm{FeCl}_{3} \cdot \mathrm{H}_{2} \mathrm{O}$} & 0 & 0,0789 & 21,12 \\
& Sem adição & 1,575 & 0,0973 & 30,83 \\
& Metade & $*$ & 0,0739 & 30,41 \\
& Normal & 6,3 & 0,1028 & 25,92 \\
& Dobro & 15,75 & 0,0741 & 17,99 \\
\hline & Quíntuplo & 0 & 0,0712 & 9,36 \\
$\mathrm{CuSO}_{4} \cdot 5 \mathrm{H}_{2} \mathrm{O}$ & Sem adição & 0,006 & 0,0736 & 18,11 \\
& Metade & $*$ & 0,0739 & 30,41 \\
& Normal & 0,024 & 0,0855 & 3,90 \\
& Dobro & 0,06 & 0,0508 & 6,56 \\
\hline & Quíntuplo & 0 & 0,0633 & 15,78 \\
& Sem adição & 0,006 & 0,0827 & 24,16 \\
& Metade & $*$ & 0,0739 & 30,41 \\
$\mathrm{CoCl}_{2} \cdot \mathrm{H}_{2} \mathrm{O}$ & Normal & 0,024 & 0,0788 & 25,36 \\
& Dobro & 0,06 & 0,04723 & 7,06 \\
\hline
\end{tabular}

* refere-se à experimento realizado em que as concentrações dos micronutrientes foram as mesmas fornecidas pela literatura, sem alterações nesta.

Um teste de reprodutibilidade utilizando as melhores concentrações de $\mathrm{FeCl}_{3} \cdot \mathrm{H}_{2} \mathrm{O}$ e $\mathrm{CoCl}_{2} \cdot \mathrm{H}_{2} \mathrm{O}$ (metade da concentração na solução-estoque) e também as condições otimizadas pelo PCC para concentração de $\mathrm{ZnSO}_{4} \cdot 7 \mathrm{H}_{2} \mathrm{O}(0,06 \mathrm{~g} / \mathrm{L})$ e $\mathrm{MnCl}_{2} \cdot 4 \mathrm{H}_{2} \mathrm{O}(0,5 \mathrm{~g} / \mathrm{L})$ foi efetuado visando confirmar deve-se trabalhar com a metade de $\mathrm{FeCl}_{3} \cdot \mathrm{H}_{2} \mathrm{O}$ e $\mathrm{CoCl}_{2} \cdot \mathrm{H}_{2} \mathrm{O}$, aliado com os pontos ótimos das concentrações de $\mathrm{ZnSO}_{4} \cdot 7 \mathrm{H}_{2} \mathrm{O}$ e $\mathrm{MnCl}_{2} \cdot 4 \mathrm{H}_{2} \mathrm{O}$. Pelo teste de reprodutibilidade foi verificado que quando há uma alteração na concentração dos reagentes $\mathrm{FeCl}_{3} \cdot \mathrm{H}_{2} \mathrm{O}$ e $\mathrm{CoCl}_{2} \cdot \mathrm{H}_{2} \mathrm{O}$ com $\mathrm{ZnSO}_{4} \cdot 7 \mathrm{H}_{2} \mathrm{O}$ e $\mathrm{MnCl}_{2} \cdot 4 \mathrm{H}_{2} \mathrm{O}$, não há melhora significativa no teor de lipídeos, sendo abaixo de 47,5\%, valor do teor de lipídeos que foi encontrado somente com a alteração em $\mathrm{ZnSO}_{4} \cdot 7 \mathrm{H}_{2} \mathrm{O}$ e $\mathrm{MnCl}_{2} \cdot 4 \mathrm{H}_{2} \mathrm{O}$, em que a biomassa esteve em 0,0966 g/L. Nesse aspecto, concluise que dentre os micronutrientes, não é viável a alteração destes quando for utilizar $0,06 \mathrm{~g} / \mathrm{L}$ de $\mathrm{ZnSO}_{4} \cdot 7 \mathrm{H}_{2} \mathrm{O}$ e $0,5 \mathrm{~g} / \mathrm{L} \mathrm{MnCl}_{2} \cdot 4 \mathrm{H}_{2} \mathrm{O}$

\subsection{Estudo da variação das concentrações das concentrações doa macronutrientes}


Os resultados para os experimentos com alterações nas concentrações dos macronutrientes e utilizando as concentrações de zinco e manganês otimizadas, são apresentados na Tabela 2.

Tabela 2 - Resultado para o teor de lipídeos e biomassa para cada experimento com alteração nas concentrações dos macronutrientes.

\begin{tabular}{ccccc}
\hline Macronutriente & Condição & $\begin{array}{c}\text { Concentração na } \\
\text { solução-estoque } \\
(\mathbf{g} / \mathbf{L})\end{array}$ & $\begin{array}{c}\text { Biomassa } \\
(\mathbf{g} / \mathbf{L})\end{array}$ & $\begin{array}{c}\text { Teor de Lipídeos } \\
\text { extraído das } \\
\text { células } \mathbf{( \% )} \text { g/g }\end{array}$ \\
\hline \multirow{2}{*}{$\mathrm{CaCl}_{2} \cdot 2 \mathrm{H}_{2} \mathrm{O}$} & Sem adição & 0 & 0,075 & 26,77 \\
& Metade & 18,38 & 0,150 & 40,79 \\
& Dobro & 73,52 & 0,225 & 7,41 \\
\hline \multirow{2}{*}{$\mathrm{MgSO}_{4}$} & Sem adição & 0 & 0,057 & 11,72 \\
& Metade & 9,02 & 0,120 & 40,49 \\
& Dobro & 36,08 & 0,138 & 36,22 \\
\hline \multirow{2}{*}{$\mathrm{NaHCO}_{3}$} & Sem adição & 0 & 0,064 & 10,47 \\
& Metade & 6,3 & 0,110 & 30,21 \\
& Dobro & 25,2 & 0,064 & 26,01 \\
\hline \multirow{2}{*}{$\mathrm{K}_{2} \mathrm{HPO}_{4} \cdot 3 \mathrm{H}_{2} \mathrm{O}$} & Sem adição & 0 & 0,001 & 0,00 \\
& Metade & 5,705 & 0,010 & 34,25 \\
& Dobro & 22,82 & 0,230 & 4,34 \\
\hline \multirow{2}{*}{$\mathrm{NaNO}_{3}$} & Sem adição & 0 & 0,127 & 39,37 \\
& Metade & 42,55 & 0,155 & 32,17 \\
& Dobro & 170,02 & 0,150 & 26,68 \\
\hline
\end{tabular}

Com base nos resultados apresentados na Tabela 2, nota-se que quando se utilizou a metade da concentração de $\mathrm{CaCl}_{2} \cdot 2 \mathrm{H}_{2} \mathrm{O}$ e metade de $\mathrm{MgSO}_{4}$ o teor de lipídeos foi próximo de ao encontrado no melhor ponto, quando se utilizou as condições otimizadas dos micronutrientes (teor de lipídeos 47,5\% e concentração de biomassa 0,0966g/L). Este fato mostra que também poderia reduzir a concentração destes macronutrientes, no mesmo meio em que já é utilizado as melhores condições dos micronutrientes. Assim, como individualmente, o $\mathrm{CaCl}_{2} \cdot 2 \mathrm{H}_{2} \mathrm{O}$ e $\mathrm{MgSO}_{4}$ quando utilizados em metade de suas concentrações, também produziram elevadas concentrações de óleo, semelhante ao ponto em que há o teor de lipídeos de 47,5\% na combinação otimizada dos micronutrientes fontes de zinco e manganês, foi efetuado um experimento combinando junto as diferentes concentrações de $\mathrm{CaCl}_{2} \cdot 2 \mathrm{H}_{2} \mathrm{O}$ e metade de $\mathrm{MgSO}_{4}$. Quando se combina as diferentes concentrações de $\mathrm{CaCl}_{2} \cdot 2 \mathrm{H}_{2} \mathrm{O}$ e $\mathrm{MgSO}_{4}$ não se consegue superar a produção de lipídeos quando o experimento é cultivado apenas com a alteração nas concentrações de $\mathrm{ZnSO}_{4} \cdot 7 \mathrm{H}_{2} \mathrm{O}$ e $\mathrm{MnCl}_{2} \cdot 4 \mathrm{H}_{2} \mathrm{O}$ (teor de lipídeos: 47,5\%). Assim, não é viável proceder com nenhuma alteração nos macronutrientes

\section{CONCLUSÕES}

Não é viável a alteração dos micro e macronutrientes do meio de cultivo da Scenedesmus sp, com exceção $\mathrm{ZnSO}_{4} \cdot 7 \mathrm{H}_{2} \mathrm{O}$ e $\mathrm{MnCl}_{2} \cdot 4 \mathrm{H}_{2} \mathrm{O}$. Para os macronutrientes: a redução para a metade 
da concentração de $\mathrm{CaCl}_{2} \cdot 2 \mathrm{H}_{2} \mathrm{O}$ e $\mathrm{MgSO}_{4}$ pode até ser viável em um determinado momento, porém só quando esta alteração é feita somente em um destes, e não simultamente.

\section{AGRADECIMENTOS}

Agradecimento ao apoio financeiro da Fundação de Amparo à Pesquisa do Estado de Minas Gerais - FAPEMIG (Processo PCE-00089-14), VALE S.A, CAPES, CNPq - Brasil e à Faculdade de Engenharia Química da Universidade Federal de Uberlândia.

\section{6 . REFERÊNCIAS BIBLIOGRÁFICAS.}

FOLCH, J; LEES, M. STANLEY, G.H.S. A simple method for the isolation and purification of total lipids from animal tissues. J. Biol. Che,v. 22, p. 497-509, 1975.

GRIS, Lara Regina Soccol. Produção de microalga Nannochloropsis oculada em fotobiorreatores Airlift. Porto Alegre, Universidade Federal de Rio Grande do Sul. 148 p. 2010.

GUILLARD, R. R. L. Culture of marine invertebrate animals. Plenum, New York, USA, p. 29-6. 1975.

LOURENÇO, S. de O. Cultivo de microalgas marinhas: princípios e aplicações. Brasil: Rima, 2006.

MUÑOZ, R. T.; KÖLLNER, C.; GUIEYSSE, B.; MATTIASSON, B. Photosynthetically oxygenated salicylate biodegradation in a continuous stirred tank photobioreactor. Biotechnology and Bioengineering. n.87. p.797-803, 2006. 